\title{
Tubular free by cyclic groups act freely on CAT(0) cube complexes
}

\author{
J. O. Button
}

\begin{abstract}
We identify when a tubular group (the fundamental group of a finite graph of groups with $\mathbb{Z}^{2}$ vertex and $\mathbb{Z}$ edge groups) is free by cyclic and show, using Wise's equitable sets criterion, that every tubular free by cyclic group acts freely on a $\mathrm{CAT}(0)$ cube complex.
\end{abstract}

2010 Mathematics Subject Classification:

Primary 20F65, 20F67; Secondary 20E08

\section{Introduction}

When dealing with finitely presented groups, it may well be the case that knowledge of how they act geometrically allows us to prove purely group theoretic facts. An extreme example of this occurs with a word hyperbolic group, because if it acts properly and cocompactly on a CAT(0) cube complex (which will therefore be finite dimensional and locally finite) we can conclude by the Agol - Wise results [17], [1] that it will be the fundamental group of a virtually special cube complex, and therefore has a finite index subgroup which embeds in a right angled Artin group (a RAAG) by [12]. In this case we say our group is virtually special and then any property that holds for all subgroups of RAAGs will hold virtually for this hyperbolic group. However hyperbolicity is certainly needed here: if we remove this condition then the Burger - Mozes groups in [6] act properly and cocompactly on a 2 dimensional CAT(0) cube complex, but they can be simple groups which will certainly not be virtually special.

In this paper we will consider two families of groups which all contain $\mathbb{Z}^{2}$ and therefore cannot be hyperbolic but which are generally considered 
to be well behaved. The first is non hyperbolic free by cyclic groups, where here free by cyclic will always mean (finite rank free) by $\mathbb{Z}$ groups. It was proved in [11] that if a free by cyclic group is word hyperbolic then it does act properly and cocompactly on a CAT(0) cube complex and so has the above strong group theoretic properties. Now if a free by cyclic group is not word hyperbolic then it contains $\mathbb{Z}^{2}$ (as shown in [2] for the irreducible case and [5] in general) so we can ask: does such a group always have a "nice" geometric action on a CAT(0) cube complex? Of course it depends on what is meant by nice but in [10] Gersten displayed a free by cyclic group that cannot act properly and cocompactly on any CAT(0) space. Moreover this group is not virtually special (see the next section).

The other class of groups we consider in this paper are what have been called the tubular groups: namely the fundamental group of a finite graph of groups with all vertex groups isomorphic to $\mathbb{Z}^{2}$ and all edge groups isomorphic to $\mathbb{Z}$. These have been considered from both a geometric and group theoretic point of view. In [3] they were shown to have interesting Dehn functions and their quasi-isometric classes were considered in [8]. However they can be badly behaved group theoretically as they need not be linear, residually finite or even Hopfian. As for the existence of nice geometric actions, the Gersten group is in fact also a tubular group, so proper and cocompact actions on a CAT(0) cube complex will not always exist. However in [18] Wise looked at the question of when a tubular group has a free action on a CAT(0) cube complex. As all our groups are torsion free and because proper here means topologically proper (a compact set can only have finitely many elements which translate it to an image that intersects this set), acting properly and acting freely mean the same thing in this context. In [18] Corollary 5.10 the tubular groups acting freely and cocompactly on a CAT(0) cube complex are classified, though these are quite restrictive. However, on removing the cocompactness hypothesis, the main result in Theorem 1.1 of that paper gives a condition (in terms of what are called equitable subsets of the vertex groups) that determines exactly when a tubular group acts freely on a CAT(0) cube complex (though this complex might not be finite dimensional, nor locally finite). From this he was able to show that a wider range of tubular groups have a free action on a $\mathrm{CAT}(0)$ cube complex, in particular his non Hopfian example in [16] and the Gersten group, but he also used this condition to give examples of tubular groups with no such actions. Afterwards in [19] a criterion for when a tubular group acts freely on a finite dimensional CAT(0) space was developed, and in the forthcoming 
paper [20] it is shown that this is equivalent to the group being virtually special.

Here we take the intersection of these two classes of groups, namely the tubular groups which are also free by cyclic. We first identify in Proposition 2.1 exactly which tubular groups are free by cyclic, which are those groups having a homomorphism to $\mathbb{Z}$ that is non zero on all edge groups. This always holds if the underlying graph is a tree. In [18], Conjecture 1.8 states that every free by cyclic group acts freely on a CAT(0) cube complex. Using such a homomorphism we then show in Theorem 2.4 that any tubular free by cyclic group satisfies Wise's equitable subsets condition and therefore does have a free action on a $\mathrm{CAT}(0)$ cube complex.

\section{Tubular free by cyclic groups act freely}

Given a finite graph of groups $G(\Gamma)$ with all vertex groups isomorphic to $\mathbb{Z}^{2}$ and all edge groups isomorphic to $\mathbb{Z}$, we can produce a presentation for the resulting fundamental group $G$, which will be referred to as a tubular group, in the usual way. We first pick a maximal tree in $\Gamma$ and contract each edge by forming an amalgamated free product. As $\mathbb{Z}^{2}$ has an obvious 2-generator 1-relator presentation and we need to add 1 relator each time when performing the amalgamation, this process creates a presentation with $2 v$ generators and $2 v-1$ relations if there are $v$ vertices. Having done this, we then introduce a stable letter for each of the $b$ edges left ( $b$ being the first Betti number of the graph $\Gamma$ ) and form HNN extensions identifying the remaining cyclic subgroups, thus resulting in a presentation for $G$ which has $2 v+b$ generators and $2 v+b-1$ relators. In particular $G$ has a presentation of deficiency 1, that is where the number of generators is 1 more than the number of relators.

Moreover any finite presentation for $G$ has deficiency at most 1, which can be seen because the standard presentation 2-complex forms an aspherical graph of spaces as in [14] Section 3. Thus $G$ is also of cohomological and geometric dimension 2 and its Euler characteristic will equal 0, where 1 minus the Euler characteristic is an upper bound for the deficiency of $G$. Also $G$ is well known to be coherent, namely every finitely generated subgroup of $G$ is finitely presented.

Another well behaved class of groups sharing these nice properties are the free by cyclic groups. Therefore it is of interest to determine when a tubular 
group is actually free by cyclic, with the next result giving us a complete answer.

Proposition 2.1 A tubular group $G$ is isomorphic to a free by cyclic group if and only if there exists a homomorphism from $G$ to $\mathbb{Z}$ which is non zero on every edge group.

Proof. We initially proved this directly by induction on the number of vertices in the graph and using [13] Corollary 1 . This states that if $G$ is a group with an amalgamated product decomposition $G=A *_{C} B$, and $\phi: G \rightarrow \mathbb{Z}$ is a homomorphism such that $\operatorname{ker}\left(\left.\phi\right|_{C}\right)$ is finitely generated and not equal to $C$, then $\operatorname{ker}(\phi)$ is finitely generated if and only if $\operatorname{ker}\left(\left.\phi\right|_{A}\right)$ and $\operatorname{ker}\left(\left.\phi\right|_{B}\right)$ are finitely generated (with a similar result for HNN extensions). The kernel is then seen to be free by standard facts, such as [15] Section I.5.5 Theorem 14 which states that a subgroup $S$ of $H=A *_{C} B$ that misses all conjugates of $C$ is a free product of a free group with factors of the form $S \cap h A h^{-1}, S \cap h B h^{-1}$ for various elements $h \in H$.

However since then the paper [9] has appeared, giving more general results as it explains how to calculate the BNS invariant of a graph of groups. In particular [9] Corollary 2.10 tells us the following. Suppose that $G$ is finitely generated and is the fundamental group of a finite graph of groups where all edge inclusions are proper. Suppose also that the vertex groups are slender (meaning that every subgroup is finitely generated). Then if $\chi: G \rightarrow \mathbb{Z}$ is a surjective homomorphism, we have that $\operatorname{ker} \phi$ is finitely generated if and only if $\chi$ is non trivial on every edge group.

In order to see that this finitely generated kernel must be free, we know that moreover it is finitely presented because $G$ is a graph of groups with coherent vertex groups (every finitely generated subgroup is finitely presented) and slender edge groups, thus $G$ itself is coherent. Therefore we can use the comment after Theorem 7.3 in [7] because $G$ has deficiency 1.

Corollary 2.2 If $\Gamma$ is a tree then $G(\Gamma)$ is free by cyclic.

Proof. We pick a vertex $v_{0}$ with which to start defining $\chi$, which for now will be a homomorphism from $G$ to $\mathbb{Q}$, and then inductively extend to the vertices at distance $n$ from $v_{0}$. Say this has been done in such a way that $\chi$ is non zero on every edge group and take a vertex $v$ at distance $n+1$. We can 
let this vertex group equal $\langle x, y\rangle$ where the edge group into $v$ from distance $n$ is generated by a power of $x$ and thus we have $\chi(x) \neq 0$. We then have other generators for the edge groups out of $v$ which will all be of the form $x^{i} y^{j}$. As this is a finite list, we can choose a value of $\chi(y) \in \mathbb{Q}$ such that no edge group in $v$ is sent to zero by $\chi$. We then extend $\chi$ to all of the vertex groups, hence to $G$, and this will be well defined because $\Gamma$ is a tree.

We end up with a homomorphism $\chi$ from a finitely generated group to $\mathbb{Q}$, thus the image is cyclic and we can multiply $\chi$ by a suitable integer so that this image now lies in $\mathbb{Z}$.

Next we examine the necessary and sufficient condition stated in [18] Theorem 1.1 for a tubular group to act freely on a CAT(0) cube complex (which in general here need not be finite dimensional nor locally finite). This condition is that there exists an equitable set, which is a choice of a finite family of elements from each vertex group such that

(i) each family generates a finite index subgroup of the respective vertex group and

(ii) the compatibility condition is satisfied on each edge $e$, namely on taking a generator of this edge group which embeds as $\mathbf{x}$ in one vertex group and $\mathbf{y}$ in the other, we have

$$
\sum_{i=1}^{m} \#\left[\mathbf{x}, \mathbf{s}_{\mathbf{i}}\right]=\sum_{j=1}^{n} \#\left[\mathbf{y}, \mathbf{t}_{\mathbf{j}}\right] .
$$

Here $\left\{\mathbf{s}_{\mathbf{1}}, \ldots, \mathbf{s}_{\mathbf{m}}\right\}$ is the chosen family for the first mentioned vertex group, $\left\{\mathbf{t}_{\mathbf{1}}, \ldots, \mathbf{t}_{\mathbf{n}}\right\}$ is for the second and $\#[\mathbf{x}, \mathbf{s}]$ is the intersection number. If we have $\mathbf{x}=\left(x_{1}, x_{2}\right)$ and $\mathbf{s}=\left(s_{1}, s_{2}\right)$ for some choice of basis of the first vertex group then \# $[\mathbf{x}, \mathbf{s}]$ is equal to the modulus of the determinant $x_{1} s_{2}-x_{2} s_{1}$ of the matrix $\left(\begin{array}{ll}x_{1} & s_{1} \\ x_{2} & s_{2}\end{array}\right)$, so that we will write $|(\mathbf{x} \mathbf{s})|$ for $\#[\mathbf{x}, \mathbf{s}]$.

It was shown in [18] Example 1.4 that the Gersten free by cyclic tubular group as in Example 3.3 satisfies the above condition, thus although it does not act properly and cocompactly on any CAT(0) metric space by [10], or even properly and semisimply by [4], it does act freely on a CAT(0) cube complex. Conjecture 1.8 of this paper says that every free by cyclic group possesses such an action. We will now confirm this conjecture for the tubular free by cyclic groups.

For the proof we will be working over $\mathbb{Q}$ rather than $\mathbb{Z}$. To this end, we 
define a "Q্Q-tubular group" $G^{\mathbb{Q}}(\Gamma)$ as follows: on taking the finite graph $\Gamma$, every vertex group will consist of a copy of $\mathbb{Q}^{2}$ (rather than $\mathbb{Z}^{2}$ as before) along with a given basis. However the edge groups will still all be copies of $\mathbb{Z}$ and we have edge inclusion maps $e_{ \pm}: \mathbb{Z} \hookrightarrow \mathbb{Q}^{2}$ for each edge $e$, where we regard $\mathbb{Z}$ and $\mathbb{Q}^{2}$ as additive groups. Thus $G^{\mathbb{Q}}(\Gamma)$ is a graph of groups with $\mathbb{Q}^{2}$ vertex groups and $\mathbb{Z}$ edge groups, so as such will have a fundamental group $G^{\mathbb{Q}}$ although we will not be interested in the group theoretic properties of $G^{\mathbb{Q}}$. Rather we can use linear algebra to find equitable sets for suitable $\mathbb{Q}$-tubular groups which are defined as follows: an equitable set for $G^{\mathbb{Q}}(\Gamma)$ is a choice at each vertex of a spanning family of elements for that vertex group $\mathbb{Q}^{2}$ such that the compatibility condition in (ii) above holds on each edge $e$ in $\Gamma$. Here we are defining $\#[\mathbf{x}, \mathbf{s}] \in \mathbb{Q}$ to be the modulus of the determinant $x_{1} s_{2}-x_{2} s_{1}$ when $\mathbf{x}=\left(x_{1}, x_{2}\right)$ and $\mathbf{s}=\left(s_{1}, s_{2}\right)$ are expressed in terms of the given basis of the appropriate vertex group $\mathbb{Q}^{2}$. (This means that at a particular vertex an arbitrary change of basis could well change the value of $[\mathbf{x}, \mathbf{s}]$ but this is well defined if we only allow basis changes with determinant \pm 1 .)

Moreover given a tubular group $G(\Gamma)$ in the original sense of having $\mathbb{Z}^{2}$ vertex groups, we obtain the associated $\mathbb{Q}$-tubular group $G^{\mathbb{Q}}(\Gamma)$ as follows: first take a $\mathbb{Z}$-basis for each vertex group $\mathbb{Z}^{2}$ and then enlarge it to a copy of $\mathbb{Q}^{2}$ such that the $\mathbb{Z}^{2}$ subgroup embeds naturally as the set of integer lattice points. We also have as a basis for $\mathbb{Q}^{2}$ the image of this $\mathbb{Z}$-basis that was chosen. Then take the same edge inclusions $e_{ \pm}: \mathbb{Z} \hookrightarrow \mathbb{Z}^{2}$ but now regard these maps as having codomain $\mathbb{Q}^{2}$. We first note a proposition relating tubular and $\mathbb{Q}$-tubular groups.

Proposition 2.3 If $G(\Gamma)$ is a tubular free by cyclic group with associated $\mathbb{Q}$-tubular group $G^{\mathbb{Q}}(\Gamma)$ then:

(a) There exists a group homomorphism $\chi^{\mathbb{Q}}$ from the fundamental group $G^{\mathbb{Q}}$ to $\mathbb{Q}$ which is non zero on the edge groups of $G^{\mathbb{Q}}(\Gamma)$.

(b) An equitable set for $G(\Gamma)$ gives rise to one for $G^{\mathbb{Q}}(\Gamma)$ and vice versa.

Proof. For (a) we use Proposition 2.1 to obtain the homomorphism $\chi: G \rightarrow$ $\mathbb{Z}$, hence $\chi: G \rightarrow \mathbb{Q}$, which is non zero on all the edge groups of $G(\Gamma)$. We can now extend $\chi$ at each vertex group $\mathbb{Q}$-linearly to obtain $\chi^{\mathbb{Q}}: G^{\mathbb{Q}} \rightarrow \mathbb{Q}$ and we still have the same edge groups. As for (b), at each vertex we have used the same $\mathbb{Z}$-basis for both our $\mathbb{Z}^{2}$ and $\mathbb{Q}^{2}$ vertex groups and the edge group inclusions are unchanged, thus for $\mathbf{x}, \mathbf{s} \in \mathbb{Z}^{2}$ the intersection number in $\mathbb{Z}^{2}$ is the same in $\mathbb{Q}^{2}$ and so an equitable set for $G(\Gamma)$ immediately becomes one 
for $G^{\mathbb{Q}}(\Gamma)$. This also works the other way except that the family of elements $\left\{\mathbf{s}_{\mathbf{1}}, \ldots, \mathbf{s}_{\mathbf{m}}\right\}$ providing the equitable set at a particular vertex will generally be elements of $\mathbb{Q}^{2}$, not $\mathbb{Z}^{2}$. But an equitable set remains equitable if every element is multiplied by the same non zero rational throughout, so we can multiply these elements by a single common denominator so that they all lie in $\mathbb{Z}^{2}$, whereupon we have an equitable set for $G(\Gamma)$ in the original sense for tubular groups.

We now come to the proof of our main theorem.

Theorem 2.4 If $G(\Gamma)$ is a tubular free by cyclic group then there exists an equitable set for $G(\Gamma)$ and so $G$ acts freely on a $C A T(0)$ cube complex.

Proof. We replace $G(\Gamma)$ with its $\mathbb{Q}$-tubular equivalent $G^{\mathbb{Q}}(\Gamma)$ throughout and find an equitable set for the latter group, which suffices by Proposition 2.3. Also by Proposition 2.3 we have $\chi^{\mathbb{Q}}: G^{\mathbb{Q}} \rightarrow \mathbb{Q}$ which is non zero on all the edge groups.

Our first case is when $\Gamma$ is a bouquet of circles, so that we have only one vertex $v$ but many self loops. Having picked a $\mathbb{Z}$-basis for the vertex group of $G(\Gamma)$, and hence for the only vertex group of $G^{\mathbb{Q}}(\Gamma)$, as well as having directed the $k$ edges arbitrarily, we suppose we have the following generators for the images of the edge groups when injected via the negative and the positive ends respectively:

$$
\mathbf{a}_{\mathbf{1}}=\left(a_{1}, b_{1}\right), \ldots, \mathbf{a}_{\mathbf{k}}=\left(a_{k}, b_{k}\right) \text { and } \mathbf{c}_{\mathbf{1}}=\left(c_{1}, d_{1}\right), \ldots, \mathbf{c}_{\mathbf{k}}=\left(c_{k}, d_{k}\right)
$$

which are all elements of $\mathbb{Q}^{2}$. We now require a choice of $N$ elements $\mathbf{x}_{1}, \ldots, \mathbf{x}_{\mathbf{N}} \in \mathbb{Q}^{2}$ such that for each $j$ with $1 \leq j \leq k$ the following equation holds:

$$
\sum_{i=1}^{N}\left|\left(\mathbf{x}_{\mathbf{i}} \mathbf{a}_{\mathbf{j}}\right)\right|=\sum_{i=1}^{N}\left|\left(\mathbf{x}_{\mathbf{i}} \mathbf{c}_{\mathbf{j}}\right)\right| .
$$

We will be able to do this with $N=2$ by proceeding as follows:

Let the generators $(1,0)$ and $(0,1)$ of our vertex group $\mathbb{Q}^{2}$ be mapped to $m$ and $n$ respectively under $\chi^{\mathbb{Q}}$, and suppose the basis has been chosen such that $m \neq 0$ (otherwise we can make a $\mathbb{Z}$-basis change which will not affect the following intersection numbers). Let $l_{j}$ be $\chi^{\mathbb{Q}}\left(\mathbf{a}_{\mathbf{j}}\right)=m a_{j}+n b_{j}$ so that 
also $l_{j}=\chi^{\mathbb{Q}}\left(\mathbf{c}_{\mathbf{j}}\right)=m c_{j}+n d_{j}$, with no $l_{j}$ being equal to 0 by the properties of $\chi^{\mathbb{Q}}$. On setting $l=l_{1}$, the idea is to consider as candidates for the $\mathbf{x}_{\mathbf{i}}$ points of the form $\left(\frac{l-n y_{i}}{m}, y_{i}\right)$ for $y_{i} \in \mathbb{Q}$ to be determined. Note that as all of these points lie on the line $m x+n y=l \neq 0$, these will span $\mathbb{Q}^{2}$ provided only that we take more than one distinct point of this form. We find for each $j$ between 1 and $k$ that

$$
\sum_{i=1}^{N}\left|\left(\mathbf{x}_{\mathbf{i}} \mathbf{a}_{\mathbf{j}}\right)\right|=\sum_{i=1}^{N}\left|\left(\begin{array}{cc}
\frac{l-n y_{i}}{m} & \frac{l_{j}-n b_{j}}{m} \\
y_{i} & b_{j}
\end{array}\right)\right|=\sum_{i=1}^{N} \frac{\left|l b_{j}-l_{j} y_{i}\right|}{|m|}=\frac{\left|l_{j}\right|}{|m|} \sum_{i=1}^{N}\left|y_{i}-l \frac{b_{j}}{l_{j}}\right|
$$

and so we also have

$$
\sum_{i=1}^{N}\left|\left(\mathbf{x}_{\mathbf{i}} \mathbf{c}_{\mathbf{j}}\right)\right|=\frac{\left|l_{j}\right|}{|m|} \sum_{i=1}^{N}\left|y_{i}-l \frac{d_{j}}{l_{j}}\right| .
$$

Now if given two $k$-tuples of rationals $\left(q_{1}, \ldots, q_{k}\right)$ and $\left(r_{1}, \ldots, r_{k}\right)$, we can find $N$ and $y_{1}, \ldots, y_{N} \in \mathbb{Q}$ such that

$$
\sum_{i=1}^{N}\left|y_{i}-q_{j}\right|=\sum_{i=1}^{N}\left|y_{i}-r_{j}\right|
$$

holds for each $j$ between 1 and $k$ by setting $N=2$, then letting $y_{1}$ be the minimum $m$ of our $2 k$ rational entries and $y_{2}$ be the maximum $M$ whereupon both sums become $M-m$ regardless of $j$. (In order to ensure that $y_{1} \neq y_{2}$ for spanning, we can increase $M$ and decrease $m$ if needed.) Thus having chosen $y_{1}, y_{2} \in \mathbb{Q}$ we know that $\mathbf{x}_{\mathbf{1}}, \mathbf{x}_{\mathbf{2}} \in \mathbb{Q}^{2}$ as above provides us with our equitable set for $G^{\mathbb{Q}}(\Gamma)$.

For a general graph $\Gamma$, we will reduce to the above case by contracting edges one by one in a maximal tree. However, on the level of graphs of groups, this will not be the usual procedure used when calculating the fundamental group. It is actually the replacement of one $\mathbb{Q}$-tubular group by another, in that given an oriented edge $e$ running from the vertex $v \in V(\Gamma)$ to the vertex $w \neq v$, the graph of groups $G^{\mathbb{Q}}(\Gamma)$ will be replaced by a graph of groups $G_{1}^{\mathbb{Q}}\left(\Gamma_{1}\right)$ where $\Gamma_{1}$ is the result of contracting the edge $e$ in $\Gamma$ onto the vertex $w$. However the fundamental groups $G^{\mathbb{Q}}$ and $G_{1}^{\mathbb{Q}}$ will in general be different. The procedure is not to amalgamate the vertex groups $G_{v}$ and $G_{w}$ but rather to replace $G_{v}$ with $G_{w}$ using a suitable isomorphism between them that respects the homomorphism $\chi^{\mathbb{Q}}$. We then reinterpret the inclusions into 
$G_{v}$ of the edge groups for the other edges ending at $v$ as inclusions into $G_{w}$ using this isomorphism.

Given the first edge $e_{1}$ to be contracted, with vertex groups $V_{1}^{+}$and $V_{1}^{-}$ both isomorphic to $\mathbb{Q}^{2}$ at the vertices $v_{1}^{+}, v_{1}^{-}$of $e_{1}$, let $g_{1}^{+}$be the injection into $V_{1}^{+}$of the generator of the edge group at $e_{1}$, and let $k_{1}^{+}$be a non zero element of $V_{1}^{+}$such that $\chi^{\mathbb{Q}}\left(k_{1}^{+}\right)=0$. Note that $\chi^{\mathbb{Q}}\left(g_{1}^{+}\right) \neq 0$ by hypothesis, so $g_{1}^{+}$and $k_{1}^{+}$span $\mathbb{Q}^{2}$. We also do the same at the other end of $e_{1}$ to obtain two elements $g_{1}^{-}$and $k_{1}^{-}$of $V_{1}^{-}$and we then define the isomorphism $\theta_{1}: V_{1}^{-} \rightarrow V_{1}^{+}$by sending $g_{1}^{-}$and $k_{1}^{-}$to $g_{1}^{+}$and $k_{1}^{+}$respectively. However, as the elements $k_{1}^{ \pm 1}$ could be changed under multiplication by a non zero scalar, we now choose them such that the matrix $P_{1}$, which represents $\theta_{1}$ with respect to the original bases chosen for the vertex groups, has determinant 1 in modulus. Moreover as $\chi^{\mathbb{Q}}\left(g_{1}^{+}\right)=\chi^{\mathbb{Q}}\left(g_{1}^{-}\right)$and $\chi^{\mathbb{Q}}\left(k_{1}^{+}\right)=\chi^{\mathbb{Q}}\left(k_{1}^{-}\right)$we have that $\chi^{\mathbb{Q}}\left(\theta_{1}\left(\lambda g_{1}^{-}+\mu k_{1}^{-}\right)\right)=\chi^{\mathbb{Q}}\left(\lambda g_{1}^{+}+\mu k_{1}^{+}\right)$on temporarily writing these groups additively.

We now contract the edge $e_{1}$ from the vertex $v_{1}^{-}$to $v_{1}^{+}$. This means that all other edges (if any) meeting $v_{1}^{-}$get moved to meet $v_{1}^{+}$and we replace each generator of the image of these edge groups in $v_{1}^{-}$by applying $\theta_{1}$ to this element. This results in the graph $\Gamma_{1}$ which is the result of contracting the edge $e_{1}$ in $\Gamma$, along with infinite cyclic edge groups and embeddings of these into the neighbouring vertex groups $\mathbb{Q}^{2}$. Note that as $\chi^{\mathbb{Q}}$ respects this substitution, we will also obtain a homomorphism $\chi_{1}^{\mathbb{Q}}$ from the new fundamental group $G_{1}^{\mathbb{Q}}$ to $\mathbb{Q}$ which is non zero on all edge groups.

We now continue this process, producing further isomorphisms $\theta_{2}, \theta_{3}, \ldots$, $\theta_{l-1}$ until $\Gamma$ has been contracted to the graph $\Gamma_{l}$ with just one vertex $v_{l}$, though there could be many self loops at this vertex. By the previous argument, we can find an equitable set $\left\{\mathbf{x}_{1}, \mathbf{x}_{2}\right\}$ consisting of a pair of elements that are both in $\mathbb{Q}^{2}$ for the labelled graph $\Gamma_{l}$. This means that for each of the $k$ edges and pair of elements $\mathbf{a}_{\mathbf{j}}, \mathbf{c}_{\mathbf{j}} \in \mathbb{Q}^{2}$ at either end of the $j$ th edge, we have

$$
\left|\left(\mathbf{x}_{1} \mathbf{a}_{\mathbf{j}}\right)\right|+\left|\left(\mathbf{x}_{\mathbf{2}} \mathbf{a}_{\mathbf{j}}\right)\right|=\left|\left(\mathbf{x}_{\mathbf{1}} \mathbf{c}_{\mathbf{j}}\right)\right|+\left|\left(\mathbf{x}_{\mathbf{2}} \mathbf{c}_{\mathbf{j}}\right)\right| .
$$

(If $\Gamma$ were a tree then we would have no edges left so in this case we would not contract the final edge. It is then straightforward to find an equitable set consisting of a pair of elements for each of the two remaining vertices.)

Now we reverse our contracting process and our isomorphisms. This means that if immediately prior to the application of the final isomorphism 
$\theta_{l-1}$, we have that there is some $j$ where $\mathbf{a}_{\mathbf{j}}$ is based at $v_{l}$ but $\mathbf{c}_{\mathbf{j}}$ is based at the other vertex $v_{l-1}$, we replace $\mathbf{c}_{\mathbf{j}}$ by the element $\theta_{l-1}^{-1}\left(\mathbf{c}_{\mathbf{j}}\right)$ so that this element now lies back in the vertex group $V_{l-1}$. However we also pick the elements $\theta_{l-1}^{-1}\left(\mathbf{x}_{\mathbf{1}}\right)$ and $\theta_{l-1}^{-1}\left(\mathbf{x}_{\mathbf{2}}\right)$ for the part of our equitable set based at $v_{l-1}$. To see this does make an equitable set, note that we already had equation (2) holding and we are now replacing (for $i=1,2) \mathbf{x}_{\mathbf{i}}$ by $P_{l-1}^{-1}\left(\mathbf{x}_{\mathbf{i}}\right)$ and $\mathbf{c}_{\mathbf{j}}$ by $P_{l-1}^{-1}\left(\mathbf{c}_{\mathbf{j}}\right)$, because $P_{l-1}^{-1}$ is the matrix representing $\theta_{l-1}^{-1}$. But for any 2 by 2 matrix $M$ and $\mathbf{x}, \mathbf{y} \in \mathbb{Q}^{2}$ we have

$$
|(M \mathbf{x} M \mathbf{y})|=|M(\mathbf{x}, \mathbf{y})|=|M||(\mathbf{x}, \mathbf{y})|
$$

and as here $M=P_{l-1}^{-1}$ has determinant \pm 1 , we see that the right hand side of (2) is unchanged when we insert the new elements. Similarly both sides of (2) are unchanged if for another value of $j$ both $\mathbf{a}_{\mathbf{j}}$ and $\mathbf{c}_{\mathbf{j}}$ get moved back to the vertex $v_{j-1}$, and if they both stay at $v_{j}$ then nothing at all in (2) is changed.

We can now reverse this process, introducing equitable sets at each new vertex until we return to $\Gamma$, which results in our original $\mathbb{Q}$-tubular group $G^{\mathbb{Q}}(\Gamma)$ but now with a pair of equitable elements at every vertex.

Example: The Gersten free by cyclic group

$$
G=\left\langle a, b, c, t \mid t a t^{-1}=a, t b t^{-1}=b a, t c t^{-1}=c a^{2}\right\rangle
$$

was famously introduced in [10] as an example of a group which does not act properly and cocompactly on a CAT(0) space. Also it is not virtually special: we thank Mark Hagen for explaining this. If there was a finite index subgroup $H$ of $G$ which was a subgroup of a RAAG (without loss of generality finitely generated because $H$ is) then this RAAG acts properly and cocompactly on the Salvetti complex, so $H$ acts properly on a finite dimensional cube complex. Then we can use quasiconvex walls obtained from $H$ to induce an action of $G$ on a CAT(0) cube complex, of higher but still finite dimension. This will also be a proper action and finite dimensionality tells us that $G$ acts properly and semisimply on this cube complex. However [4] points out that Gersten's result extends to proper and semisimple actions on a CAT(0) space, thus giving us a contradiction. The Gersten group does act freely and hence properly on a CAT(0) cube complex by [18] but this will necessarily be infinite dimensional. 
On rewriting this presentation as

$$
\left\langle a, b, c, t \mid[a, t], b^{-1} t b=a t, c^{-1} t c=a^{2} t\right\rangle
$$

we see that, on setting $t=u$ and $a=v$, we have a tubular group with one vertex and two self loops, where the vertex group is $\langle u, v\rangle$, the stable letter $b$ conjugates $u$ to $u v$ and the stable letter $c$ conjugates $u$ to $u v^{2}$. We now follow the proof of Theorem 2.4 in this case to obtain the homomorphism $\chi$ which sends $u$ to 1 and $v$ to 0 . On taking $u, v$ as our ordered basis for $\mathbb{Z}^{2}$ and for $\mathbb{Q}^{2}$ we have

$$
\mathbf{a}_{1}=(1,0), \mathbf{a}_{2}=(1,0), \mathbf{c}_{1}=(1,1), \mathbf{c}_{2}=(1,1)
$$

with $m=1, n=0$ and $l=l_{1}=1=l_{2}$. Thus we are looking for $\mathbf{x}_{1}=\left(1, y_{1}\right)$ and $\mathbf{x}_{2}=\left(1, y_{2}\right)$, where we will need to ensure that $y_{1} \neq y_{2}$, and satisfying both equations

$$
\left|y_{1}\right|+\left|y_{2}\right|=\left|y_{1}-1\right|+\left|y_{2}-1\right| \text { and }\left|y_{1}\right|+\left|y_{2}\right|=\left|y_{1}-2\right|+\left|y_{2}-2\right| .
$$

Then the proof tells us that we can take $y_{1}=0$ and $y_{2}=2$ to obtain $\left\{\mathbf{x}_{\mathbf{1}}=(1,0), \mathbf{x}_{\mathbf{2}}=(1,2)\right\}$, which is indeed an equitable set. An equitable set also of two elements was obtained in [18] Example 1.4 for the Gersten group, using a slightly different presentation, and on rewriting the presentation back in this form it is indeed the same equitable set.

\section{References}

[1] I. Agol, The virtual Haken conjecture, Doc. Math. 18 (2013) 1045-1087.

[2] M. Bestvina and M. Feighn, A combination theorem for negatively curved groups, J. Differential Geom. 35 (1992) 85-101.

[3] N. Brady and M. R. Bridson, There is only one gap in the isoperimetric spectrum, Geom. Funct. Anal. 10 (2000) 1053-1070.

[4] M. R. Bridson and A. Haefliger, Metric spaces of non-positive curvature, Springer-Verlag, Berlin, 1999.

[5] P. Brinkmann Hyperbolic automorphisms of free groups, Geom. Funct. Anal. 10 (2000) 1071-1089. 
[6] M. Burger, S. Mozes, Finitely presented simple groups and products of trees, C. R. Acad. Sci. Paris Ser. I Math. 324 (1997), no. 7, 747-752.

[7] J. O. Button, Large groups of deficiency 1, Israel J. Math. 167 (2008) 111-140.

[8] C.H. Cashen, Quasi-isometries between tubular groups, Groups Geom. Dyn. 4 (2010) 473-516.

[9] C. H. Cashen and G. Levitt, Mapping tori of free group automorphisms, and the Bieri-Neumann-Strebel invariant of graphs of groups, http://arxiv.org/1412.8582

[10] S. M. Gersten, The automorphism group of a free group is not a CAT(0) group, Proc. Amer. Math. Soc. 121 (1994) 999-1002.

[11] M.F. Hagen and D. T. Wise, Cubulating hyperbolic free-by-cyclic groups: the general case, Geom. Funct. Anal. 25 (2015) 134-179.

[12] F. Haglund and D. T. Wise, Special cube complexes, Geom. Funct. Anal. 17 (2008) 1551-1620.

[13] J.G. Ratcliffe, On normal subgroups of an amalgamated product of groups with applications to knot theory, Boletin de la Sociedad Matematicatica Mexicana 20 (2014) 287-296.

[14] G. P. Scott and C. T.C. Wall, Topological Methods in Group Theory. Homological group theory (Proc. Sympos., Durham, 1977), pp. 137-203, London Math. Soc. Lecture Note Ser., 36, Cambridge Univ. Press, Cambridge-New York, 1979.

[15] J.-P. Serre, Trees, Springer-Verlag, Heidelberg, 1980.

[16] D. T. Wise, A Non - Hopfian Automatic Group, J. Alg. 180 (1996) 845847.

[17] D. T. Wise, From Riches to Raags: 3-Manifolds, Right-Angled Artin Groups, and Cubical Geometry, CBMS Regional Conference Series in Mathematics No. 117, American Mathematical Society, Providence, RI, 2012. 
[18] D. T. Wise, Cubular tubular groups, Trans. Amer. Math. Soc. 366 (2014) $5503-5521$.

[19] D. J. Woodhouse, Classifying finite dimensional cubulations of tubular groups, http://arxiv.org/1502.02619

[20] D. J. Woodhouse, Classifying virtually special tubular groups, to appear.

Selwyn College, University of Cambridge, Cambridge CB3 9DQ, UK

E-mail address: j.o.button@dpmms.cam.ac.uk 